\title{
Épopée et décadence
}

\author{
Jean-Louis Backès \\ Université de Paris Sorbonne
}

Qu'est-ce que l'épopée?

À l'âge classique, on proposait de superbes définitions, toutes plus vagues les unes que les autres. Nous n'avons guère changé sur ce point. À l'âge classique, on n'hésitait pas à compléter la définition, quelle qu'elle soit, par un exemple, par un exemple pour ainsi dire exemplaire, singulier. L'épopée, c'était l'Enéide et tout ce qui, de près ou de loin, lui ressemblait. Cette manière de penser pourrait être qualifiée de « mythique ». Par opposition aux pensées de la raison, qui manient des catégories générales, la pensée mythique joue du singulier. 
Aujourd'hui, l'Énéide a un peu perdu de sa prééminence. C'est une épopée parmi d'autres. Elle ne fournit pas la définition du genre. De plus, nous nous rappelons la célèbre parole de Saussure : "C'est une mauvaise méthode que de partir des mots pour définir les choses. » (1968, p. 31.) Le mot « épopée » a une longue histoire, pleine d'aventures. Il présente d'innombrables implications, qui ne sont pas toujours toutes réalisées, tant s'en faut.

\section{Implications du mot « épopée »}

L'exemple de l'Énéide mettait en évidence une implication importante : l'épopée racontait l'origine de la nation. Au XVIe siècle déjà, au moment où se formaient les grands États européens, au moment où l'on inventait des normes pour les langues nationales, on souhaitait qu'un grand poème célèbre la terre des ancêtres. Ronsard tente, et manque, la Franciade. Camoens réussit les Lusiades. Le Tasse va plus loin: il fait l'épopée de la chrétienté. Le modèle virgilien est toujours présent.

Une des grandes vertus de l'Énéide est de réunir en un seul texte les deux poèmes majeurs de la tradition grecque: Énée voyage en mer, comme l'Ulysse de l'Odyssée; il fait la guerre, comme l'Achille de l'Iliade. Virgile a imité Homère; nul n'en doute. C'est même une de ses grandes vertus. Non seulement il offre un modèle, le modèle, à quiconque veut faire une épopée à la gloire de son propre pays, mais encore il montre que l'imitation est possible : ce qu'il a fait avec le texte grec, on peut le faire avec le sien. 
Une question se pose, pourtant: les poèmes homériques n'évoquent pas l'origine de la nation grecque, à supposer que cette expression ait un sens dans la haute antiquité. Ulysse est un roi parmi beaucoup d'autres. Les Grecs, certes, sont unis contre les Troyens, mais les Troyens, qui parlent grec, qui ont des noms grecs, sont aussi admirables que les guerriers d'Argos ou de Thessalie. La chute de Troie, annoncée dans l'Iliade, n'est pas l'acte de fondation d'un État grec qui n'a jamais existé.

La remarque a son importance. Si «épopée » implique «célébration des origines de la nation », ni l'Iliade ni l'Odyssée ne sont à proprement parler des épopées.

Mais si l'on s'en tient au mot "origine», les choses se présentent de manière un peu différente. Laissons le mot se diluer dans un mot plus vaste. "Épopée » implique "passé ». C'est vrai dans un grand nombre de cas. Le Tasse a théorisé la chose : il faut que se soient écoulés trois ou quatre cents ans entre l'événement que l'on célèbre et celui qui le célèbre. Chose curieuse : ce délai est celui qui sépare la date présumée de la chute de Troie et la date présumée de la naissance d'Homère. C'est aussi - et Le Tasse pouvait ne pas le savoir — la longueur de l'intervalle qui sépare la mort du Roland historique et la mise par écrit de La Chanson de Roland. L'épopée dit des histoires d'autrefois.

Naturellement, cette implication n'est pas toujours réalisée : les Lusiades évoquent en 1572 un héros qui est mort en 1524. Hermann und Dorothea, que les Allemands classent volontiers parmi les épopées, évoque un événement tout récent. L'Énéide chante un passé très lointain, mais elle célèbre aussi le présent: le héros, aux Enfers, aperçoit la foule des grands hommes à venir, y compris les contemporains du poète. Rome 
persiste dans sa grandeur initiale. Aucune allusion n'est faite à sa possible décadence, même provisoire.

Il n'en va pas de même dans toutes les épopées. Le passé est parfois l'objet d'une nostalgie. Parmi les grands guerriers de l'Iliade figure un noble vieillard, qui se souvient; on a parfois l'impression que pour Nestor, roi de Pylos, «les jeunes d'aujourd'hui ne valent pas les vieux», comme on chantait dans une parodie des Burgraves. Indépendamment de ce personnage, on peut songer à l'une de ces formules dont la maîtrise faisait les aèdes: plusieurs fois un combattant, oubliant les armes classiques, lance et épée, soulève une pierre pour écraser son adversaire, une pierre telle que «deux hommes d'aujourd'hui » (Iliade, v. 303) ne pourraient pas la soulever.

Ces minces détails rencontrent une grande idée, qui a inquiété plus d'un poète, à l'âge classique: les héros de l'épopée, qu'ils soient ou non fils de dieux, ne cessent, dans Homère et dans Virgile, de rencontrer sur leur route des Immortels. Ils appartiennent à un autre âge que le nôtre. C'était l'avis des premiers auditeurs, ou des premiers lecteurs, qui avaient renoncé, pour leur part, à voir les Olympiens face à face. C'était l'avis des admirateurs, quinze ou vingt siècles plus tard : ces dieux-là n'existaient pas; ils appartenaient au domaine de la fiction, du conte, du «il était une fois». De toute façon, ils relevaient de la merveille, voire du miracle. Mais le grand Pan est mort, et il n'y a presque plus de miracles.

Le monde épique est-il un monde perdu? On pourrait parfois le croire, et que le mot « épopée » implique, en bien des cas, le mot « décadence». 


\section{Survie de l'épopée}

Mais si le monde que décrit l'épopée est un monde voué à la décadence, l'idée même d'écrire une épopée, depuis quelque temps, est en voie de se perdre : c'est l'épopée elle-même qui est en décadence.

Une formule abusivement célèbre proclame que «les Français n'ont pas la tête épique ». Il n'est pas nécessaire de la discuter; elle n'a presque aucun sens. Il suffira de rappeler qu'elle a été mise à la mode par la préface de La Henriade, que ce poème a passé, pendant quelques décennies, pour la réalisation enfin réussie de l'Énéide française et qu'il $a$, très rapidement, sombré comme beaucoup d'autres. On connaît la terrible remarque de Chateaubriand: "Si un plan sage, une narration vive et pressée, de beaux vers, une diction élégante, un goût pur, un style correct, sont les seules qualités nécessaires à l'Épopée, La Henriade est un poème achevé. Mais cela ne suffit pas. » $(1966$, p. 238)

Chateaubriand lui-même n'a pas mieux fait. Et ses Martyrs se sont presque évanouis, à l'époque où l'on retrouvait La Chanson de Roland. N'y a-t-il donc d'épopées que dans les premiers âges de l'humanité?

Une fois de plus, on a l'impression que l'épopée appartient à un âge mythique, à un âge où les héros étaient plus grands que nous, à un âge où les dieux visitaient en personne la terre. L'épopée ne se contente pas d'évoquer cet âge; elle lui appartient presque tout entière. Elle ne peut vivre qu'à son ombre.

Le XIXe siècle reste marqué par cette image. Malgré tout, certains de ses poètes n'hésitent pas à reprendre la grande 
route de l'imitation virgilienne. On le voit à un indice: la formule du prélude est constante.

Arma virumque cano [...] (Virgile, Énéide)

Of Man's First disobedience [...]

Sing Heav'nly Muse Milton (John Milton, Paradise Lost)

Je chante ce héros qui régna sur la France

Et par droit de conquête et par droit de naissance [...]

(Voltaire, La Henriade)

Cante uno chato de Prouvènço.

Dins lis amour de sa jouvènço,

A travès de la Crau, vers la mar, dins li bla,

Umble escoulan dóu grand Oumèrou

Iéu la vole segui1 . [...] (Frédéric Mistral, 1978, p. 52)

La formule consacrée est bien en place. Elle sera suivie de l'invocation à la divinité :

Tu, Segnour Diéu de ma patrio,

Que nasquères dins la pastriho,

Enfioco mi paraulo et douno-me d'alen²! (p. 54)

La fidélité à la tradition homérique, par-delà Virgile, est affirmée de la manière la plus nette. Mais la volonté de servir la patrie ne l'est pas moins. Ce n'est pas qu'il soit question de dire les origines de la nation. Le monument est construit à la gloire de la Provence - y a-t-il une nation provençale? - et de sa langue, de "nosto lengi mespresado», de "notre langue méprisée». Il n'est pas question de héros, de batailles, de

\footnotetext{
1 « Je chante une jeune fille de Provence. - Dans les amours de sa jeunesse, à travers la Crau, vers la mer, dans les blés, - humble écolier du grand Homère, - je veux la suivre. » La traduction française est due à Mistral luimême.

2 «Toi, Seigneur Dieu de ma patrie, - qui naquis parmi les pâtres, enflamme mes paroles et donne-moi du souffle! — »
} 
voyages sur la mer. L'héroïne est une jeune fille de la campagne; le héros est un vannier.

Et pourtant. On n'a pas lu six pages que maître Ambroise se met à chanter. Il chante la chanson du bailli de Suffren, qui est parti sur la mer pour défaire les Anglais. Le bailli, «lou Baile», est né près d'Aix-en-Provence; il embarque à Toulon; ses équipages sont provençaux. Après sa victoire, il va rendre compte au roi de France, son maître, et nul ne l'a plus revu. La chanson est ancienne.

- E vaqui, quand Marto fielavo,

Li cansoun, dis, que se cantavo!

Eron bello, o jouvènt, e tiravon de long...

L'er s'es fa'n pau vièi, mais que provo ?

Aro n'en canton de pu novo,

En franchimand ${ }^{3}[\ldots]$ (p. 70)

Voilà que revient l'antique implication: quelque chose s'est perdu depuis les temps héroïques. Certainement, l'épopée n'est pas seule en cause. Tous les admirateurs de Nerval pensent à Sylvie, à la manière dont elle désapprend le chant des aïeules : elle phrase.

Mais revenons à cette expression qu'un francophone pourrait ne pas comprendre : «quand Marthe filait». Mistral met une note, pour rappeler que Sainte Marthe a terrassé à Tarascon le monstre, la tarasque, et s'est alors établie près de la ville. En fait, les connotations sont plus riches encore : Marthe est celle qui agit, pendant que Marie écoute parler le Christ. De

3 « — "Et voilà, quand Marthe filait, — les chansons, dit-il, que l'on chantait! — Elles étaient belles, ô jouvenceaux, et tiraient en longueur... - L'air a un peu vieilli, mais qu'importe? - Maintenant on en chante de plus nouvelles, - en français [...]" » 
toute façon, par la grâce d'un simple nom propre, on est renvoyé aux temps jadis, à l'époque des miracles.

$\mathrm{Ne}$ nous y trompons pas: le récit de Mistral est constamment réaliste; tous les événements qui se produisent relèvent du vraisemblable; nombre de descriptions portent sur l'activité quotidienne d'un domaine rural. Et pourtant...

\section{Réalisme et merveilleux}

Notons que le poète n'a pas dit de son poème qu'il était une épopée. Il s'est contenté du prélude et de la division en douze chants, comme dans l'Énéide.

Auguste Brizeux s'y est pris de manière encore plus discrète. S'il a prévu vingt-quatre chants, comme dans l'Iliade ou l'Odyssée, il a renoncé aux formules du prélude. Ce n'est pas qu'il évite le ton solennel.

J'entends au loin, j'entends les landes s'éveiller! Au murmure des flots lasses de sommeiller, Les paroisses d'Arvor veulent que je les nomme ; Merlin dans son tombeau triomphe d'un long somme : Dormez encor, Merlin! O Bretagne, pourquoi, Quand le monde inquiet partout marche sans loi, Pêcheurs, sur vos îlots, pâtres, sous vos ramures, Solitaires manoirs, pourquoi tous ces murmures? Où les prendre ces chants que vous me demandez? Silence, ô mers de l'ouest! l'esprit souffre, attendez! » (Brizeux, p. 99)

Le poète a choisi la langue française, alors qu'il est capable d'écrire en breton. Il a choisi l'alexandrin à rimes plates, consacré à l'épopée par la tradition la plus sévère. N'a-t-on pas dit que Ronsard avait manqué son œuvre pour s'être limité au 
décasyllabe? Mistral, peut-être plus attentif à la tradition italienne, avait préféré une strophe complexe, et tout à fait originale.

Quelques indices suffisent. Les lecteurs ont reconnu l'épopée, ou tout au moins la tentation épique. Et certains d'entre eux se vexent. Ce n'est pas là exactement ce qu'on leur avait appris dans les écoles. «Un juge très autorisé a exprimé le regret qu'un poëme intitulé les Bretons ne fût pas consacré surtout à la Bretagne héroïque des DuGuesclin et des Beaumanoir, des Montfort et des Clisson. » (Saint-René Taillandier, "Notice sur Brizeux», dans Brizeux, p. XL). SaintRené Taillandier précise que le «juge autorisé » est Charles Magnin et renvoie à la Revue des Deux Mondes du 1er août 1845.

Gustave Planche pose la question autrement: «M. Brizeux a-t-il voulu faire un poëme? » $(1855$, p. 735) Évoquant un passage de LaGuerre des Gaules, il demande si le poète « n'eût pas trouvé dans le journal militaire de Jules César l'étoffe d'une admirable épopée» (p. 749). Il pense aussi à Dom Lobineau, bénédictin, auteur d'une mémorable Histoire de Bretagne (1707). Il regrette que Brizeux ait «négligé avec le même dédain ces deux sources fécondes » (p. 750). Comme, de plus, à son avis, Les Bretons se présentent comme une suite d'épisodes assez peu liés les uns aux autres, il conclut que «le livre est plutôt un roman qu'un poëme » (p. 750).

On voit la puissance des préjugés. Une épopée devrait s'appuyer sur l'histoire, sur le passé lointain, seul héroïque. Que reproche-t-on en fait à Brizeux? Peut-être d'avoir, quelques années avant Mistral (Les Bretons paraissent en 1845 ; Mirèio, en 1858) chanté «un jeune paysan» et une «fille» toute 
simple. Ces gens-là ont droit tout au plus au roman : Manzoni, après d'autres, l'a montré, dans ses Promessi Sposi.

Il y a pourtant des exemples. Hermann und Dorothea est sans doute une "idylle», dont les héros sont des gens du peuple. Goethe a d'ailleurs, pour les mettre en scène, renoncé à l'honorable hexamètre et retenu un vers plus léger. Cela ne signifie pas pour autant qu'il ait fait choix de personnages médiocres. Il raconte à Eckermann, le 23 mars 1829, qu'un ami lui a reproché d'avoir laissé l'héroïne prendre des armes pour se défendre. "Mais sans ce trait le caractère de cette extraordinaire jeune fille, tel qu'il convient au temps et aux circonstances, est détruit, et elle tombe au niveau de l'ordinaire. » (Eckermann, p. 466 ; je traduis).

C'est sur l'aspect exceptionnel, « extraordinaire » de ces héros sans illustration que repose toute la question.

\section{Les héros et les traditions}

Je voudrais montrer que Brizeux et Mistral ont tous les deux cherché à justifier la haute stature de leurs héros et de leurs héroïnes non seulement par leurs vertus propres, qui sont des vertus relativement banales, mais par la relation très particulière qu'ils et elles entretiennent avec une tradition encore vivante, quoique en train de s'éteindre.

«Ta couronne est tombée, antique souveraine ». (Brizeux, p. 100). Privé de contexte, ce vers n'est pas clair. Qui désigne-til? La duchesse Anne, dont le mariage avec un roi de France a fait oublier l'indépendance de son domaine? Une Bretagne allégorique, sacrifiée sur l'autel du jacobinisme? Le contexte 
offre une autre solution : on entend un peu plus tôt un appel à une «vivante harmonie ». Est-ce l'harmonie du paysage breton, évoqué en quelques vers ? Est-ce l'harmonie en elle-même, à laquelle Brizeux a consacré de nombreux poèmes à tendance philosophique ? L'idée de décadence est là, malgré tout.

Il faut aller plus loin. Tournons la page. Voici une cérémonie, un «pardon », une procession.

Les croix marchaient devant; sur un riche brancard,

Couverte d'un manteau de soie et de brocart,

La Vierge de Coad-Rî suivait, blanche et sereine,

Le front couronné d'or comme une jeune reine.

(Brizeux, p. 101)

La madone serait-elle une autre forme de l'«antique souveraine »? Il faut d'abord noter une nette opposition, dans les vers qui suivent, entre l'humble ferveur des paysans et la piété confortable de ceux que le poète appelle «nous ». Ce sont les gens des villes qui sont par là clairement désignés, ceux qui vont

Souhaitant qu'à l'autel le prêtre abrège l'heure,

Et tout bas regrettant l'aise de leur demeure. (p. 101)

Le poète se considère-t-il comme l'un des leurs? Rien n'est moins sûr. En tout cas, pendant quelque temps, on peut avoir l'impression que l'épopée oppose un pays profond encore religieux à une capitale où la civilisation a réduit la religion à un système de convenances formelles. Quelle religion? Le christianisme, sans doute.

N'allons pas aussi vite. Rappelons-nous que la question du merveilleux païen ou chrétien a fait se déchirer les donneurs de leçons, il n'y a pas si longtemps, à propos de l'épopée. Pour peindre les Bretons de son temps, Brizeux ne peut pas ne pas 
évoquer le culte chrétien. Il ne peut pas ne pas mentionner les " incrédules», dont le nombre augmente de jour en jour. Il ne faut pourtant pas s'étonner de le voir, au troisième chant, reporter plus loin ce schéma d'opposition.

La harpe a disparu. Notre terre est sans voix.

Nous ne savons plus rien des hommes d'autrefois.

(Brizeux, p. 117)

Le poème raconte alors l'histoire de la Bretagne depuis les plus lointains commencements, jusqu'au jour où

Tout fut soumis au Christ, et, signe triomphant,

La croix sanctifia la pierre du Peûlvan. (p. 120)

Le développement est fait à la troisième personne. Dans le chant cinquième, c'est un personnage qui a pris la parole.

Notre pays s'en va! tout décline, tout passe!

Grand Dieu! pour renverser nos usages bénis,

Avec les cœurs sans foi les prêtres sont unis. (p. 133)

Un clergé éclairé a laissé tomber en désuétude des rites qu'il tient pour de pure superstition : on ne bénit plus les vaches au pardon de Carnac; saint Cornéli n'est plus supposé les protéger. Autrefois,

Tous vivaient confiants, les hommes et les bêtes ;

Et les jours de Pardons, m'assurait mon aïeul,

Lorsqu'on n'y menait pas son bœuf, il venait seul. » (p. 133)

Mais un autre personnage intervient :

Alors un étranger : « Vos pères et leurs prêtres

Eux-mêmes n'ont-ils pas oublié leurs ancêtres.

Dans le champ où ses bœufs ont tracé leur sentier,

Le char de Cornéli passa-t-il le premier?

Hu-Cadarn est-il donc mort dans votre mémoire? » (p. 134) 
On peut avoir quelques doutes sur l'authenticité des doctrines antiques dont Brizeux se fait ici l'écho. Y croit-il? Non, sans doute. Ce qui l'intéresse, c'est la continuité qu'il observe entre les traditions: le christianisme, aujourd'hui en décadence, a repris des images, des récits, des gestes qui lui sont bien antérieurs. L'idée de revenir à ces rituels disparus n'a pas de sens. Il importe seulement qu'ils ne soient pas ignorés. L'étranger qui les fait revivre par la seule parole révèle finalement son identité.

- Eh! pourquoi

Ne vous dirais-je pas, Bretons, que c'était moi ? (p. 135)

Il vaut la peine de remarquer un phénomène étrange. Les critiques classiques n'ont pas tort: le poème de Brizeux est assez décousu, non seulement parce que les épisodes se succèdent sans vraiment s'enchaîner, comme les étapes du voyage d'Ulysse dans l'Odyssée, mais chacun d'eux est à peine développé. Brizeux manque d'ampleur; son lecteur peut avoir l'impression d'être toujours poussé en avant, de ne pas avoir le loisir de s'arrêter, de méditer. Mais ce tempo précipité est au service d'un souci d'informer, de faire connaître mille détails, tous plus précieux les uns que les autres, et ces détails construisent justement une continuité. Et l'on se retrouve, dans cette histoire toute moderne, en relation avec des dieux depuis longtemps évanouis. Il a suffi, peut-être, de ce léger décalage entre un village un peu arriéré et une ville fière de sa civilisation neuve; il a suffi par ailleurs que, de cette ville où se trouvent les livres, un homme revienne dans le pays de son enfance pour que le mot de "présent» se mette légèrement à vaciller. "Un présent n'existe pas», dira, probablement dans un autre sens, Mallarmé. 
Les légendes de Provence se trouvent aussi, très souvent, en relation avec la très ancienne christianisation du pays. Sainte Marthe, évoquée au détour d'un vers, grâce à une expression pittoresque, joue un rôle essentiel dans la tradition. Le chant onzième de Mirèio fait raconter, par les Saintes elles-mêmes, au cours d'un rêve qui visite l'héroïne, comment elles sont venues de Judée en Provence, comment elles ont fait s'évanouir les rituels sauvages, la sorcellerie, les bêtes immondes. Tout le sixième chant était déjà consacré à l'évocation de la sorcellerie. Or, toute cette fantasmagorie est résolument moderne: c'est Mireille qui conduit Vincent blessé voir la Taven, qui connaît les anciens secrets. Mistral écrit là une manière de descente aux enfers, qui rappelle le chant onze de l'Odyssée et, plus encore, le sixième chant de l'Enéide. Brizeux avait trouvé, pour se livrer à une imitation analogue, un autre prétexte: la description des mines de Huelgoat. L'antre de la sorcière est aussi réel, aussi contemporain, que les galeries

Où l'argent et l'étain, et le cuivre et le plomb,

Le quartz et le mica se suivent en filons. (p. 202)

Il faut remarquer que l'action de la sorcière est parfaitement efficace : vilainement blessé, Vincent retrouve la santé.

Il faut observer aussi que, entre les pratiques littéralement immémoriales, qui survivent dans les légendes de fées, et les récits à demi fantastiques sur des personnages historiques qui ont réellement connu le Christ, s'intercalent, en Provence, qui est la Provincia Romana, les images d'une mythologie plus familière, même si personne n'y croit plus. Mais est-ce bien de croyance qu'il s'agit?

Dans la légende des Saintes, il est question de briser les idoles, et la Vénus d'Arles est confondue. Mais quand elle arrive 
à Tarascon pour combattre un monstre plus primitif, les gens demandent à Marthe, avec les paroles même d'Ulysse à Nausicaa :

— Quau siés ? la cassarello Diano? [...]

O Minervo la casto e la forto? ${ }^{4}$ (Mistral, p. 410)

Ce qui est ici en cause ne relève pas de la théologie; dans les derniers moments du paganisme, on a beaucoup réfléchi sur la nature des dieux; on les a accablés de significations allégoriques; on les a réduits en concepts. C'est d'une scène qu'il est ici question : la noble attitude d'un corps de femme suscite l'admiration. À la supplication d'Ulysse répond, dans l'Iliade, l'apparition d'Hélène sur le rempart.

Aristote l'a dit, il y a longtemps: l'épopée, comme la tragédie, met en scène des êtres humains qui sont «meilleurs » que la moyenne. Il avait, pour sa part, tendance à comprendre ses propres paroles en leur reconnaissant un sens qui intéresse la sociologie: ne pouvait être les meilleurs que ceux qui appartenaient aux grandes familles. Ses commentateurs, particulièrement à la Renaissance et à l'âge classique, ont surtout insisté sur le sens moral du mot; et souvent ils s'étonnaient qu'à côté du vertueux Énée, Achille le violent et Ulysse le menteur puissent faire figure de héros.

\section{Des êtres simplement admirables}

Plus d'un écrivain romantique a fait litière de ces préjugés; les uns ont choisi leurs protagonistes parmi les gens simples;

\footnotetext{
$4_{«}$ "Qui es-tu? la chasseresse Diane?" [...] — "ou Minerve la chaste et la forte?" »
} 
d'autres ont évité de faire des leurs des parangons de vertu. Quelques poètes, poètes épiques, en vers ou en prose, ont cherché, dans le monde, des êtres simplement admirables, admirables d'abord par la beauté de leur attitude, des êtres qui font penser à ces fictions disparues : les innombrables dieux des innombrables paganismes. Ils ont su que les êtres admirables sont par définition en danger. La décadence les menace, ou la mort.

Certains ont médité sur la mort des dieux. Mistral a laissé mourir Mirèio. Brizeux avait, de loin, déifié Marie. C'était son premier recueil. Il l'avait appelé d'abord, bien qu'il fût en vers, «roman». Bien vite, il s'en est repenti. Le roman est ce qui guette l'épopée affaiblie. Le roman, sous-genre de l'épopée, est la décadence de l'épopée, comme il est la décadence du mythe.

Le mot «épopée » est pour ainsi dire bardé d'implications. Quelles sont celles qui sont devenues irréalisables ? Quelles sont celles qui tiennent toujours? Il y a beaucoup à réfléchir sur le mot de Goethe, ausserordentlich, que l'on peut traduire par "extraordinaire» ou encore par «hors normes ». Comment une jeune fille toute simple peut-elle être « extraordinaire»? Hissée par Brizeux sur un autel tout poétique, Marie, la vraie Marie, disait: «Monsieur Auguste a toujours aimé rire.» Elle ne comprenait pas qu'elle était une héroïne épique. Et c'est justement parce qu'elle ne le comprenait pas qu'elle accédait à la grandeur de Mireille, qui est aussi celle de Nausicaa. 


\section{Bibliographie}

BrizeuX, Auguste. (1861), OEuvres complètes, Paris, Michel Lévy.

Chateaubriand, François-René de. (1966 [1802]), Génie du christianisme, Paris, Garnier-Flammarion.

EcKermann, Johann Peter. (1956 [1836]), Gespräche mit Goethe, Berlin, Aufbau-Verlag.

MistRAL, Frédéric. (1978 [1858]), Mireille, Paris, GarnierFlammarion.

Planche, Gustave. (1855), "Poètes et romanciers modernes de la France. Auguste Brizeux ». Revue des Deux Mondes. 2e série, t. 9, janvier-mars, p. 735-757.

SAuSSURE, Ferdinand de. (1968 [1916]), Cours de linguistique générale. Paris, Payot.

\section{Résumé}

Les Bretons de Brizeux (1845) et Mirèio (Mireille) de Mistral (1858) ont été considérés comme des épopées au sens traditionnel du terme. On n'y trouve pas cependant plusieurs des traits essentiels du genre : les personnages n'appartiennent à aucun monde aristocratique et leurs aventures n'ont aucun caractère héroïque. Mais, comme dans les grandes épopées grecques et romaines, le poète tisse dans son histoire nombre d'allusions aux légendes du passé et exprime sa nostalgie d'un monde perdu. La décadence est un thème épique; comme telle, elle joue un rôle important dans les deux poèmes. 


\begin{abstract}
Brizeux's Les Bretons (1845) and Mistral Mirèio have been considered as epics in the traditional meaning of the word. However some important features of the literary genre cannot be found in these poems: the protagonists do not belong to any aristocracy and their adventures are deprived of any heroic character. But, as in the great Greek or roman epics, the poet twistes in his story numerous allusions to the legends of the past and appears nostalgic of a lost world. Decadence is an epic theme, and plays as such an important role in both poems.
\end{abstract}

